\title{
Cálculo y diseño de instalaciones aisladas de energía solar fotovoltaica en la región central del Perú
}

\section{Calculation and design of isolated installations of photovoltaic solar energy in the central region of Peru}

\author{
José Mendoza R.", Raúl Sánchez P." y Héctor Torres (") \\ (I)Facultad de Ingeniería Eléctrica y Electrónica, Universidad Nacional del Centro del Perú \\ Email: ingjomero@yahoo.es
}

\section{Resumen}

El objetivo principal del presente trabajo de investigación es hacer que los interesados logran las competencias necesarias para realizar los cálculos y diseño correcto de una instalación aislada de energía solar fotovoltaica, como es de determinar el consumo de energía de una instalación; periodo de diseño y factor de irradiación; dimensionar el generador, determinar las capacidades del acumulador, el regulador y el inversor y la configuración de la instalación.

Para cumplir con el objetivo de cálculo y diseño de una instalación aislada de energía solar fotovoltaica en la región central del Perú se ha elaborado una hoja de cálculo en Excel para hallar el número de módulos totales necesarios, potencia total a instalar, número de ramas en paralelo, número de módulos necesarios por rama, potencia por rama, tensión por rama, capacidad nominal del acumulador, tensión nominal del acumulador, número de baterías en serie, intensidad máxima a soportar por regulador en la línea de generador, intensidad máxima a soportar por regulador en la línea de consumo y potencia nominal mínima del inversor.

Palabras clave: energía solar fotovoltaica, cálculo y diseño de instalación aislada, región central del Perú

\begin{abstract}
The main objective of this research is to make stakeholders achieve the necessary skills to perform the calculations and correct design of an isolated installation of photovoltaic solar energy, such as determining the energy consumption of an installation; factor design period and irradiation; sizing the generator, determine the capabilities of the accumulator, controller and inverter configuration and installation.

To meet the objective of calculation and design of an isolated installation of photovoltaic solar energy in the central region of Peru has developed a spreadsheet in Excel to find the total number of modules required, total power installed, number of branches in parallel, number of modules required for branch power per branch, voltage branch nominal battery capacity, nominal battery voltage, number of batteries in series, maximum support by regulator line generating current, maximum intensity to bear by regulator in the consumer line and minimum rated power inverter.
\end{abstract}

Keywords: photovoltaic solar energy, calculation and installation design isolated, central region of Peru 


\section{Introducción}

El presente trabajo de cálculo y diseño de instalaciones aisladas de energía solar fotovoltaica ha sido diseñado especialmente para la región central de nuestro país. En él se tratan los aspectos teóricos y prácticos básicos de la tecnología fotovoltaica, utilizando un lenguaje sencillo para la comprensión de cualquier tipo de personas.

Cumpliendo con el objetivo principal se ha elaborado un software amigable y sencillo para el cálculo y diseño de un sistema solar fotovoltaico aislado, además de colocar a disposición de los interesados e conocimiento básico acerca de los fundamentos de la tecnología fotovoltaica a través de un enfoque práctico del tema, desarrollando los puntos más relevantes del aspecto teórico. De este modo, los interesados podrán adquirir conocimientos acerca de la tecnología fotovoltaica, sus posibilidades, restricciones y aplicaciones, además serán capaces de diseñar pequeños sistemas fotovoltaicos aislados.

\section{Materiales y métodos}

El material usado en el presente trabajo como se había previsto es el software Excel, con él se realiza los cálculos para el diseño de una Instalación Aislada de Energía Solar Fotovoltaica en la Región Central del Perú. Para obtener los resultados primeramente se debe obtener los datos climatológicos y geográficos, estimar las cargas diarias previstas en la instalación, calcular la radiación prevista en la ubicación seleccionada, calcular la potencia necesaria de la instalación y con ellos obtendremos mediante una hoja de cálculo elaborada en Exce los resultados deseados.

\section{Resultados}

Mediante un ejemplo se explica el diseño de una instalación aislada de energía solar fotovoltaica en la ciudad de Huancayo utilizando la el software elaborado en Excel que le llamaremos "Ciaes".

Primeramente se hace clic en el cuadro que tiene un triángulo negro del recuadro lugar, en el tendremos la opción de las nueve capitales de provincias de la región Junín, elegimos Huancayo y con ello automáticamente se aparece su latitud y longitud y su radiación mínima de cada mes del año y el menor valor de radiación del mes crítico. Toda la información obtenida está ubicada en el una hoja del "Ciaes" para las nueve capitales de provincias, si se desea información de otro lugar incluso que no son de la región Junín se puede obtener su latitud y longitud por medio de Google Earth disponible en Internet y en base a esta información la radiación del lugar acudiendo a la página web de la NASA https://eosweb.larc.nasa.gov/cgi-bin/sse/grid.cgi?email=, y esta información lo puede cargar a la base de datos del
"Ciaes".

Radiación solar del lugar a ubicar el sistema de inclinación de $\mathrm{II}^{\circ}-12^{\circ}$ del panel (recomendado para la zona central del Perú)

\begin{tabular}{ccccc}
\multicolumn{1}{c}{ LUGAR } & \multicolumn{5}{c}{ HUANCAYO } \\
\hline Latitud & -12.07 & \\
\hline Longitud & -75.21 & & \\
\hline Mes & Ene & Feb & Mar & Abr \\
\hline $\begin{array}{c}\text { Radiación inclinación del } \\
\text { panel I1 }-12^{\circ} \text { kwh/(m² día) }\end{array}$ & 4.59 & 4.63 & 4.71 & 5.21 \\
\hline $\begin{array}{c}\text { Menor valor de radiación del } \\
\text { mes crítico }\end{array}$ & 4.59 & & & \\
\hline
\end{tabular}

Figura I Radiación solar del lugar.

Seguidamente se rellena el cuadro de cálculo de cargas ubicado en la hoja de CARGAS del "Ciaes". En la figura 2, se puede observar el cuadro para el ejemplo.

\section{Calculo de cargas}

\begin{tabular}{|c|c|c|c|c|c|}
\hline $\begin{array}{l}\text { U } \\
\text { ni } \\
\text { d. }\end{array}$ & Carga & $\begin{array}{l}\text { Potencia } \\
\text { Unitaria } \\
\text { (Watt) }\end{array}$ & $\begin{array}{l}\text { Horas de } \\
\text { funciona } \\
\text { miento al } \\
\text { Día }\end{array}$ & $\begin{array}{c}\text { Total } \\
\text { Energía } \\
\text { necesari } \\
\text { a (Wh) }\end{array}$ & $\begin{array}{c}\text { Total Energía } \\
\text { necesaria } \\
\text { (Wh) - Margen } \\
\text { Seguridad 20\% }\end{array}$ \\
\hline \multicolumn{6}{|c|}{ CONSUMO EN CORRIENTE DIRECTA } \\
\hline \multirow[t]{2}{*}{5} & $\begin{array}{l}\text { Lámp } \\
\text { aras } \\
\text { (DC) }\end{array}$ & 15.00 & 5.00 & 375.00 & 4450.00 \\
\hline & Total & 15 & & & \\
\hline \multicolumn{5}{|c|}{$\begin{array}{l}\text { Consumo medio de energía diaria de cargas en } \\
\text { corriente directa }\left(L_{m d, c D}\right)\end{array}$} & 4450.00 \\
\hline \multicolumn{6}{|c|}{ CONSUMO EN CORRIENTE ALTERNA } \\
\hline 1 & $\begin{array}{l}\text { Lavad } \\
\text { ora } \\
\text { (AC) }\end{array}$ & 350.00 & 1.50 & 525.00 & 630.00 \\
\hline \multirow[t]{2}{*}{1} & $\begin{array}{l}\text { Calefa } \\
\text { cción } \\
\text { (AC) }\end{array}$ & 110.00 & 10.00 & 1100.00 & 1320.00 \\
\hline & Total & 460 & & & \\
\hline \multicolumn{5}{|c|}{$\begin{array}{c}\text { Consumo medio de energía diaria de cargas en } \\
\text { corriente alterna }\left(L_{\mathrm{md}, \mathrm{CA}}\right)\end{array}$} & 1950.00 \\
\hline
\end{tabular}

Figura 2. Calculo de cargas.

Finalmente elegimos el tipo de panel solar a usar (BSI80S5 de Brisban para el ejemplo) haciendo clic en el triángulo negro del cuadro del recuadro Marca del "Ciaes" y automáticamente aparecen los valores de las características del panel solar elegido.

\section{DATOS DEL PANEL SOLAR}

\begin{tabular}{|c|c|c|c|}
\hline MARCA & \multicolumn{3}{|c|}{ BRISBAN BSI80S5 } \\
\hline Modelo & \multicolumn{3}{|c|}{ BSI80S5 } \\
\hline Potencia nominal & $\operatorname{Pmax}(\mathrm{Wp})$ & $W_{p}$ & 180 \\
\hline Tolerancia & Tol & $\%$ & 0 \\
\hline Tensión nominal & Vmpp & $\vee$ & 36.55 \\
\hline Corriente nominal & Impp & $A$ & 4.900 \\
\hline Configuración & - & $\vee$ & 24 \\
\hline Isc & Isc & $A$ & 5.3 \\
\hline Voc & Voc & $\vee$ & 44.5 \\
\hline
\end{tabular}


Figura 3. Datos del panel solar a utilizar

El "Ciaes" tiene la opción de agregar en su base de datos las características de otros paneles solares.

Después de haber ingresado los datos de carga, radiación mínima del lugar del sistema y características del panel solar a utilizar, el "Ciaes" nos da los resultados del cálculo

\begin{tabular}{|c|c|c|}
\hline \multicolumn{3}{|l|}{ CALCULO DE LOS ELEMENTOS DEL SISTEMA } \\
\hline Consumo medio de energía diario $\left(\mathrm{L}_{\mathrm{md}}\right)$ & 2755 & Wh/día \\
\hline Consumo de energía medio $\left(Q_{a h}\right)$ & 114.8 & Ah/día \\
\hline Consumo total anual ( $\left.\mathrm{L}_{\mathrm{T}}\right)$ & 1005575 & Wh/año \\
\hline Consumo medio anual ( $\left.\mathrm{L}_{\mathrm{ma}}\right)$ & 2755 & Wh/día \\
\hline Número total de paneles necesarios $\left(\mathrm{N}_{\mathrm{T}}\right)$ & 4 & \\
\hline Número de paneles en serie ( $\mathrm{N}_{\text {SERIE }}$ ) & 1 & \\
\hline Número de paneles en paralelo (Nparalelo) & 4 & \\
\hline $\begin{array}{l}\text { Corriente de generación fotovoltaico } \\
\left(I_{G F V, M P P}\right)\end{array}$ & 25.01 & \\
\hline Número de paneles en paralelo (Nparalelo) & 5 & OK \\
\hline $\begin{array}{l}\text { Capacidad nominal de la batería en función } \\
\text { de la descarga máxima diaria }\left(C_{n d}\right)\end{array}$ & 18367 & Wh \\
\hline $\begin{array}{l}\text { Capacidad nominal de la batería en función } \\
\text { de la descarga máxima diaria }\left(C_{n d}\right)\end{array}$ & 765.3 & Ah \\
\hline $\begin{array}{l}\text { Capacidad nominal de la batería en función } \\
\text { de la descarga máxima estacional }\left(C_{n e}\right)\end{array}$ & 23614.3 & Wh \\
\hline $\begin{array}{l}\text { Capacidad nominal de la batería en función } \\
\text { de la descarga máxima estacional }\left(C_{n e}\right)\end{array}$ & 983.9 & Ah \\
\hline Corriente de entrada al regulador (l entrada $)$ & 34 & A \\
\hline Corriente de salida al regulador ( $\left.I_{\text {salida }}\right)$ & 26 & A \\
\hline Potencia del inversor ( $\left.\mathrm{P}_{\text {inv }}\right)$ & 552 & W \\
\hline
\end{tabular}

Figura 4. Resultados del sistema aislado de generación de electricidad a través de radiación solar.

\section{Discusión}

1. En el Perú todavía no se tiene normas completas para este tipo de cálculos y diseños, el presente trabajo se basa generalmente a normas internacionales. Sería bueno tener cuanto antes normas que sean para nuestro país.

2. En cuanto a algunos paneles solares comerciales que existe en nuestro país no se tiene una información completa de algunas de sus características, es por ello que nos limitamos a aquellos paneles que tienen la información completa.

3. De igual modo en el caso de radiación mínima del mes crítico de nuestra región Junín, la información que se encuentra a cargo de los organismos nacionales no se encuentra actualizada, se sugiere que ya es tiempo que deben actualizarla así como lo tienen la NASA.

\section{Conclusiones}

I. Con la hoja de cálculo elaborado en Excel es posible hallar el número de módulos totales necesarios, potencia total a instalar, número de ramas en paralelo, número de módulos necesarios por rama, potencia por rama, tensión por rama, capacidad nominal del acumulador, tensión nominal del acumulador, número de baterías en serie, intensidad máxima a soportar por regulador en la línea de generador, intensidad máxima a soportar por regulador en la línea de consumo y potencia nominal mínima del inversor.

2. Los cálculos de un prototipo ha demostrado que la energía fotovoltaica aislada es una opción viable para comunidades de la región central que no tienen acceso a redes eléctricas.

3. Para realizar los cálculos de los elementos que constituyen el sistema aislado se debe tener los datos geográficos del lugar (latitud y longitud), potencia de las cargas de corriente directa y corriente alterna, radiación para el lugar elegido, y datos relativos al panel solar elegido para el sistema fotovoltaico.

\section{Referencias}

I. Méndez Muñiz, Javier María - Cuervo García, Rafael. Energía Solar Fotovoltaica. Madrid, España. Editorial Fundación Confemental, 2012.

2. Pareja Aparicio, Miguel. Energía Solar Fotovoltaica: Calculo de una Instalación Aislada. Barcelona, España. Editorial Marcombo S. A., 2010.

3. Fernández Barrera, Manuel. Energía Solar: Electricidad Fotovoltaica. Madrid, España. Editorial Liberfactory, 2010.

4. D’Addario, Mlguel. Energía Solar Fotovoltaica: Usos, Aplicaciones y Diseño. Madrid, España. Editorial Lulu.com, 2014.

5. Moro Vallina, Miguel. Instalaciones Solares Fotovoltaicas. Madrid, España. Editorial Paraninfo, 2010.

6. Alonso, Manuel. Energía Solar Fotovoltaica. Madrid, España. Editorial Pujol \& Amado S L L, 201 l.

7. Orbegozo, Carlos - Arivilca, Roberto. Energía Solar Fotovoltaica: Manual Técnico para Instalaciones Domiciliarias. Editorial Green Energy. 